\title{
A Case of Obstructive Sleep Apnea Syndrome Presenting as Paradoxical Insomnia
}

\author{
Hoyoung An \\ Seockhoon Chung \\ Department of Psychiatry, \\ University of Ulsan College of Medicine, \\ Asan Medical Center, \\ Seoul, Korea
}

A 63-year-old female with obstructive sleep apnea syndrome (OSAS) presented with clinical features indistinguishable from paradoxical insomnia (PI). Her main complaint was chronic insomnia. Her subjective sleep latency was $2-3 \mathrm{~h}$, subjective sleep time was less than $3 \mathrm{~h}$, despite spending $8 \mathrm{~h}$ in bed, and she reported near constant awareness of her surroundings while lying in bed. Her body mass index (BMI) was $22.67 \mathrm{~kg} / \mathrm{m}^{2}$, and her neck circumference was 34.5 $\mathrm{cm}$. Nocturnal polysomnography (NPSG) findings indicated severe OSAS. Her total sleep time (TST) was $359 \mathrm{~min}$, sleep latency $13 \mathrm{~min}$, and her apnea/hypopnea index (AHI) was $74.6 / \mathrm{h}$. The aim of this report is to evaluate the association between PI and OSAS cases confirmed by NPSG.

Psychiatry Investig 2010;7:75-78

Key Words Sleep apnea syndrome, Paradoxical insomnia, Sleep state misperception, Nocturnal polysomnography.

Received: September 30, 2009 Revised: November 15, 2009 Accepted: January 24, 2010

Available online: February 8, 2010

\section{Introduction}

Paradoxical insomnia (PI) is a baffling condition in which the patient complains of almost no sleep for a duration that is physiologically improbable. Furthermore, a large discrepancy between the patient's subjective complaint and a bed partner's objective report may raise doubts about the accuracy of the patient's perception. Most people suffering from insomnia tend to overestimate sleep onset latency (SOL) and underestimate total sleep time (TST) ${ }^{1-4}$; thus, the reliability of PI as an independent clinical entity has been the subject of much debate. ${ }^{5-8}$ However, the second edition of the International Classification for Sleep Disorders (ICSD-2) now recognizes PI as a separate disorder and subcategory of insomnia (Table 1). ${ }^{9}$

The diagnosis of obstructive sleep apnea syndrome (OSAS) is more straightforward. OSAS is characterized by the repeated cessation or a major reduction in upper airway airflow during sleep. It is diagnosed and quantified using the apnea/hypopnea index (AHI), the average number of apnea/hypopnea incidents during an hour as measured by nocturnal polysomnography (NPSG). AHI values of 5, 15, and 30 represent the cut-off values for mild, moderate, and severe OSAS, respectively. ${ }^{10}$ The risk factors for OSAS are well understood, and snoring is highly associated with the disorder. ${ }^{10}$ However, recent reports have suggested that the standard diagnostic indicators may not always be applicable to older adults. ${ }^{11-13}$

We present the case of a female with OSAS who presented with clinical features indistinguishable from PI. The aim of this report is to evaluate the association between PI and OSAS confirmed by NPSG.

\section{Case}

A 63-year-old female presented with chronic subjective insomnia. She was $1.51 \mathrm{~m}$ tall and weighed $51 \mathrm{~kg}$. Her body mass index (BMI) was $22.67 \mathrm{~kg} / \mathrm{m}^{2}$, and her neck circumference was $34.5 \mathrm{~cm}$. She did not have retrognathia, micrognathia, or any other significant ab- 
Table 1. ICSD-2 diagnostic criteria for paradoxical insomnia

A. The patient's symptoms meet the criteria for insomnia

B. The insomnia is present for at least one month

C. One or more of the following criteria apply:

i. The patient reports a chronic pattern of little or no sleep most nights with rare nights during which relatively normal amounts of sleep are obtained

ii. Sleep-log data during one or more weeks of monitoring show an average sleep time well below published age-adjusted normative values, often with no sleep at all indicated for several nights per week, typically there is an absence of daytime naps following such nights

iii. The patient shows a constant mismatch between objective findings from polysomnography or actigraphy and subjective sleep estimates derived either from self-report or a sleep diary

D. At least one of the following is observed

i. The patient reports constant or near constant awareness of environmental stimuli throughout most nights

ii. The patient reports a pattern of conscious thoughts or rumination throughout most nights while maintaining a recumbent posture

E. The daytime impairment reported is consistent with that reported by other insomnia subtypes, but it is much less severe than expected given the extreme level of sleep deprivation reported; there is no report of intrusive daytime sleep episodes, disorientation, or serious mishaps due to marked loss of alertness or vigilance, even following sleepless nights

F. The sleep disturbance is not better explained by another current sleep disorder, medical or neurological disorder, mental disorder, medication use, or substance use disorder.

ICSD-2: International Classification for Sleep Disorders

normality in her craniofacial features. The patient had a psychiatric history of recurrent mild depressive episodes, with full interepisodic recovery, that had started 15 years ago.

Her presenting symptoms began forty days earlier. Her subjective sleep latency was 2-3 $\mathrm{h}$ and subjective sleep time was less than $3 \mathrm{~h}$, despite spending $8 \mathrm{~h}$ in bed. She reported near constant awareness of her surroundings during most of the time she was lying in bed. She claimed her symptoms were fairly constant and that she was tired all day. She expressed concern about her sleeping problems. Nevertheless, her daughter reported that the patient's observed sleep latency was less than $30 \mathrm{~min}$, her sleep time was over $6 \mathrm{~h}$, and that the patient snored mildly.

In short, the patient fulfilled the ICSD-2 criteria for PI and, as she had no OSAS risk factors, was diagnosed with PI. A 'gold standard' treatment for PI does not yet exist, and the patient was started on a benzodiazepine/nonbenzodiazepine sedative regimen consisting of $3 \mathrm{mg}$ bromazepam, $0.25 \mathrm{mg}$ clonazepam, and $12.5 \mathrm{mg}$ zolpidem. The patient did not respond to these medications. To control underlying depression that may have accompanied her insomnia, we prescribed 15 mg mirtazapine, which has been shown to effectively control insomnia in older patients. ${ }^{14,15}$ She still showed no response, and we added $25 \mathrm{mg}$ of quetiapine, which has been reported to control insomnia. ${ }^{16}$ The patient received concurrent cognitive-behavioral therapy (CBT), and despite good compliance, her symptoms were not alleviated. After a month, the patient was admitted to the open ward. During admission, we observed that she slept quite well, though she would not acknowledge this and continued to complain of having less than $3 \mathrm{~h}$ of sleep on most nights. She snored continuously, but mildly. A change of her medication regimen to $50 \mathrm{mg}$ chlorpromazine, $200 \mathrm{mg}$ quetiapine, $100 \mathrm{mg}$ trazodone, and $1 \mathrm{mg}$ clonaz- epam had no effect. We arranged a NPSG to investigate other possible causes for her insomnia.

Although her subjective TST was under $3 \mathrm{~h}$ on the night of the test, the objective TST was $359 \mathrm{~min}$, SOL was $13 \mathrm{~min}$, and her wake-time after sleep onset (WASO) was just over $71 \mathrm{~min}$. Her sleep efficiency was $81.0 \%$. She showed almost no slowwave sleep. Stages N1, N2, and rapid eye movement (REM) occupied $16.2 \%, 54.9 \%$, and $23.4 \%$ of her TST, respectively, and REM sleep latency was $79 \mathrm{~min}$. Her AHI was $74.6 / \mathrm{h}$, indicating severe OSAS. Her minimum $\mathrm{SaO}_{2}$ level was $76.0 \%$, and her average oxygen saturation level was $92.8 \%$. Her oxygen desaturation index, which indicates the average number of oxygen desaturations $4 \%$ or more below the baseline level per hour, was 55.1/h (Figure 1, Table 2).

The NPSG results revealed that the patient had severe OSAS, and we concluded that OSAS was the cause of her insomnia. She was informed about the NPSG results and OSAS. We recommended continuous positive airway pressure, but she refused. Her symptoms of PI subsequently improved.

\section{Discussion}

PI is a complaint of severe and chronic insomnia with no objective evidence of sleep disturbance or the daytime impairment that would be expected from such an extreme degree of sleep loss. ${ }^{9}$ Patients often report extensive awareness of the environment while trying to sleep. Associated features include major concerns about sleeping problems and an unwillingness to acknowledge objective evidence indicating normal sleep patterns and duration. NPSG and the multiple sleep latency test rarely detect significant sleep disturbances in these patients. The etiology of PI is unknown, but PI subjects have been demonstrated to consume more oxygen than 
Figure 1. Hypnogram and sleep architecture of the patient. REM: rapid eye movement stage, $\mathrm{S} 1$ : sleep stage $\mathrm{N} 1$, S2: sleep stage N2, S3: sleep stage N3, LM: limb movement, PLM: periodic limb movement, Desat: oxygen desaturation, SpO2: oxygen saturation, Obstructive: obstructive apnea, Central: central apnea, Mixed: mixed apnea.

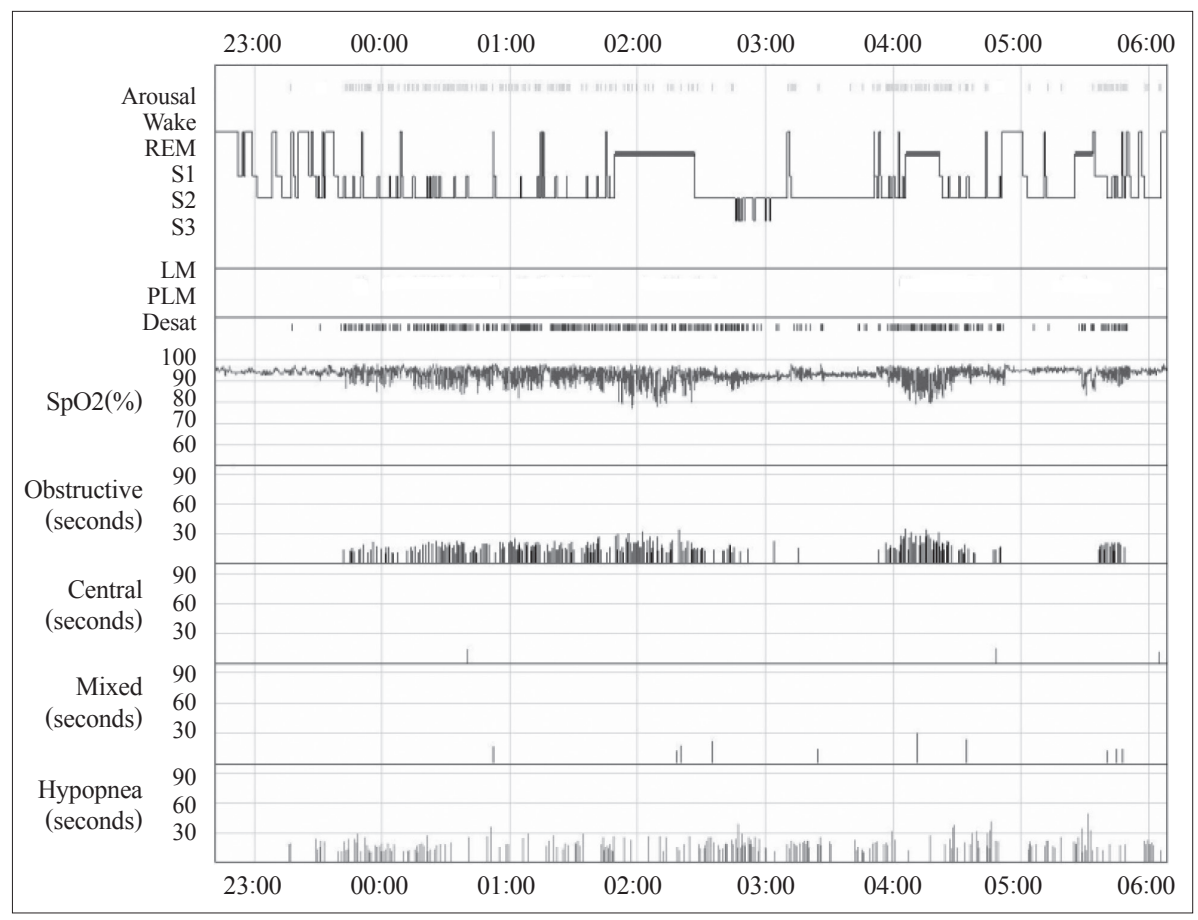

Table 2. Polysomnographic findings of the patient

\begin{tabular}{lc}
\hline \multicolumn{1}{c}{ Variables } \\
\hline Time In Bed (TIB) & $443 \mathrm{~min}$ \\
Total Sleep Time (TST) & $359 \mathrm{~min}$ \\
Sleep efficiency & $81.0 \%$ \\
Wake time After Sleep Onset (WASO) & $71 \mathrm{~min}$ \\
Sleep Onset Latency (SOL) & $13 \mathrm{~min}$ \\
Stage REM Latency & $79 \mathrm{~min}$ \\
Stage N1 & $16.2 \%$ \\
Stage N2 & $54.9 \%$ \\
Stage N3 & $5.6 \%$ \\
Stage R & $23.4 \%$ \\
Apnea Hypopnea Index (AHI) & $74.6 / \mathrm{h}$ \\
Respiratory-related Arousal Index (RAI) & $57.5 / \mathrm{h}$ \\
Spontaneous arousal index & $3.5 / \mathrm{h}$ \\
PLM arousal index & $0.0 / \mathrm{h}$ \\
Average oxygen saturation & $92.8 \%$ \\
Lowest oxygen saturation & $76.0 \%$ \\
Oxygen Desaturation Index (ODI) & $55.1 / \mathrm{h}$ \\
PSQI & 13 \\
ESS & 0 \\
\hline
\end{tabular}

REM: rapid eye movement, PLM: Periodic Limb Movement, ESS: Epworth Sleepiness Index, PSQI: Pittsburg Sleep Quality Questionnaire

do normal control subjects. This finding suggests that high levels of physiological activation may be associated with PI. ${ }^{17}$ Furthermore, a recent study compared cyclic alternating pat- terns (CAP) in people with PI and normal controls and reported that CAP were more frequent in people with PI, suggesting that repeated arousal may underlie the disorder. ${ }^{18}$

OSAS is a major cause of daytime sleepiness. Common symptoms include snoring, nocturnal snorting, and gasping. ${ }^{19}$ OSAS is strongly associated with obesity $\left(\geq 25 \mathrm{~kg} / \mathrm{m}^{2}\right)^{10,20,21}$ and old age ( $>65$ years $)^{10,20}$ and less highly associated with male gender, ${ }^{20,21}$ increased neck circumference $(\geq 39 \mathrm{~cm}),{ }^{10,21}$ a positive family history, ${ }^{10,20}$ alcohol ingestion, ${ }^{10}$ and certain craniofacial features such as retrognathia and micrognathia. ${ }^{10,20}$ Clinical symptoms in conjunction with an AHI $\geq 5 / \mathrm{h}$ are used to diagnose OSAS. The prevalence of OSAS in insomniacs has been reported to be as high as $67 \%$, but reports concerning the characteristics of insomniacs with OSAS are scarce. A few studies have indicated that sleep maintenance insomnia is the most frequent symptom reported by insomniacs with OSAS, possibly caused by repeated apnea during sleep. ${ }^{22}$ In contrast, physiological hyperarousal has been proposed to explain sleep-onset insomnia in insomniacs with OSAS. ${ }^{23}$ However, both studies used subjects suspected to have OSAS; thus, their usefulness for diagnostic differentiation between insomnia and OSAS may be limited.

The patient in the present case report fulfilled the diagnostic criteria for PI and had none of the common risk factors for OSAS. She complained of chronic insomnia with a subjective TST of less than $3 \mathrm{~h}$ despite contrary observations from family members and medical staff. She claimed she was aware of her surroundings for most of the night. NPSG revealed an AHI of 74.6/h and a respiratory arousal index, the number of arousals due to respiratory incidents per hour, of 
57.5/h; thus, she was diagnosed with severe OSAS.

Research during the past decade has demonstrated that the characteristics of OSAS in older adults differ from those of younger people. A previous study that screened a group of older insomniacs using NPSG reported that after excluding all the subjects with OSAS risk factors, the prevalence of OSAS was $29-49 \%$, depending on the cut-off value used (AHI $\geq 15$ or 5 for mild or moderate OSAS, respectively). ${ }^{13}$ Moreover, a study comparing older people with OSAS and older insomniacs reported that BMI values and the presence of snoring were similar in both groups, ${ }^{12}$ and a study comparing the clinical features of older men and young and middleaged subjects with OSAS reported that older OSAS subjects snored less, had lower BMI, smaller neck circumference, and milder daytime sleepiness. ${ }^{11}$

Sleep maintenance insomnia, the most common insomnia symptom experienced by people with OSAS, may be explained by repeated arousal during sleep, ${ }^{22}$ and repeated arousal indicated by increased CAP is a possible cause of PI. ${ }^{18}$ Sleep-onset insomnia in patients with OSAS has been hypothesized to be caused by physiological hyperarousal, similar to the increased physiological activation proposed to underlie PI. We suggest that frequent arousal caused by increased respiratory incidents may mimic the repeated arousal that occurs in PI, and physiological hyperarousal may imitate increased physiological activation, thus causing the patient to confuse sleep and wake states.

In conclusion, these findings suggest that it is more difficult to diagnosis OSAS in older people than in younger patients. In some cases, OSAS in older patients may be misdiagnosed as another sleep disorder such as PI. The factors suspected to cause insomnia in people with OSAS are similar to those proposed to cause PI, and this may further hinder differentiation between the two disorders. Currently, the only universally approved diagnostic tool for OSAS is NPSG. Thus, we suggest that older adults presenting with features similar to PI should be examined for signs of OSAS, and a NPSG should be conducted for a definitive diagnosis. Further investigation of the precise indications for NPSG in older adults with complaints of insomnia is necessary.

\section{REFERENCES}

1. Carskadon MA, Dement WC, Mitler MM, Guilleminault C, Zarcone VP, Spiegel R. Self-reports versus sleep laboratory findings in 122 drug-free subjects with complaints of chronic insomnia. Am J Psychiatry 1976;133:1382-1388.

2. Edinger JD, Fins AI. The distribution and clinical significance of sleep time misperceptions among insomniacs. Sleep 1995;18:232-239.

3. McCall WV, Turpin E, Reboussin D, Edinger JD, Haponik EF. Subjective estimates of sleep differ from polysomnographic measurements in obstructive sleep apnea patients. Sleep 1995;18:646-650.

4. Silva GE, Goodwin JL, Sherrill DL, Arnold JL, Bootzin RR, Smith T, et al. Relationship between reported and measured sleep times: the sleep heart health study (SHHS). J Clin Sleep Med 2007;3:622-630.

5. Reynolds CF 3rd, Kupfer DJ, Buysse DJ, Coble PA, Yeager A. Subtyping DSM-III-R primary insomnia: a literature review by the DSM-IV Work Group on Sleep Disorders. Am J Psychiatry 1991;148:432-438.

6. Trinder J. Subjective insomnia without objective findings: a pseudo diagnostic classification? Psychol Bull 1988;103:87-94.

7. Edinger JD, Krystal AD. Subtyping primary insomnia: is sleep state misperception a distinct clinical entity? Sleep Med Rev 2003;7:203214.

8. McCall WV, Edinger JD. Subjective total insomnia: an example of sleep state misperception. Sleep 1992;15:71-73.

9. The international classification of sleep disorders: diagnostic and coding manual (2nd ed). West-chester, IL: American Academy of Sleep Medicine; 2005.

10. Kryger MH, Roth T, Dement WC. Principles and Practice of Sleep Medicine (4th ed). Philadelphia: Elsevier Saunders; 2005.

11. Chung S, Yoon IY, Lee CH, Kim JW. Effects of age on the clinical features of men with obstructive sleep apnea syndrome. Respiration 2009; 78:23-29.

12. Endeshaw Y. Clinical characteristics of obstructive sleep apnea in community-dwelling older adults. J Am Geriatr Soc 2006;54:1740-1744.

13. Lichstein KL, Riedel BW, Lester KW, Aguillard RN. Occult sleep apnea in a recruited sample of older adults with insomnia. J Consult Clin Psychol 1999;67:405-410.

14. Bain KT. Management of chronic insomnia in elderly persons. Am J Geriatr Pharmacother 2006;4:168-192.

15. Wiegand MH. Antidepressants for the treatment of insomnia: a suitable approach? Drugs 2008;68:2411-2417.

16. Wiegand MH, Landry F, Brückner T, Pohl C, Veselý Z, Jahn T. Quetiapine in primary insomnia: a pilot study. Psychopharmacology (Berl) 2008;196:337-338.

17. Bonnet MH, Arand DL. Physiological activation in patients with Sleep State Misperception. Psychosom Med 1997;59:533-540.

18. Parrino L, Milioli G, De Paolis F, Grassi A, Terzano MG. Paradoxical insomnia: the role of CAP and arousals in sleep misperception. Sleep Med 2009;10:1139-1145.

19. Kales A, Cadieux RJ, Bixler EO, Soldatos CR, Vela-Bueno A, Misoul CA, et al. Severe obstructive sleep apnea--I: Onset, clinical course, and characteristics. J Chronic Dis 1985;38:419-425.

20. Banno K, Kryger MH. Sleep apnea: clinical investigations in humans. Sleep Med 2007;8:400-426.

21. Young T, Shahar E, Nieto FJ, Redline S, Newman AB, Gottlieb DJ, et al. Predictors of sleep-disordered breathing in community-dwelling adults: the Sleep Heart Health Study. Arch Intern Med 2002;162:893900

22. Chung KF. Insomnia subtypes and their relationships to daytime sleepiness in patients with obstructive sleep apnea. Respiration 2005;72: 460-465.

23. Gold AR, Gold MS, Harris KW, Espeleta VJ, Amin MM, Broderick JE. Hypersomnolence, insomnia and the pathophysiology of upper airway resistance syndrome. Sleep Med 2008;9:675-683. 